\begin{tabular}{|c|c|}
\hline Title & Effect of initial densities in the lattice Boltzmann model for non-ideal fluid with curved interface \\
\hline Author(s) & Gong, Jiaming; Oshima, Nobuyuki \\
\hline Citation & $\begin{array}{l}\text { Physics of fluids, 29(6), } 67108 \\
\text { https://doi.org/10.1063/1.4989638 }\end{array}$ \\
\hline Issue Date & $2017-06-28$ \\
\hline Doc URL & http:/hdl.handle.net/2115/70861 \\
\hline Rights & $\begin{array}{l}\text { This article may be downloaded for personal use only. Any other use requires prior permission of the author and A IP } \\
\text { Publishing.The following article appeared in Physics of Fluids } 29,067108 \text { (2017) and may be found at } \\
\text { http://aip.scitation.org/doi } / 10.1063 / 1.4989638 \text {. }\end{array}$ \\
\hline Type & article \\
\hline File Information & 1.4989638.pdf \\
\hline
\end{tabular}

Instructions for use 


\section{Effect of initial densities in the lattice Boltzmann model for non-ideal fluid with curved interface}

Jiaming Gong and, and Nobuyuki Oshima

Citation: Physics of Fluids 29, 067108 (2017); doi: 10.1063/1.4989638

View online: http://dx.doi.org/10.1063/1.4989638

View Table of Contents: http://aip.scitation.org/toc/phf/29/6

Published by the American Institute of Physics

\section{Articles you may be interested in}

Three-dimensional simplified and unconditionally stable lattice Boltzmann method for incompressible isothermal and thermal flows

Physics of Fluids 29, 053601 (2017); 10.1063/1.4983339

High-order regularization in lattice-Boltzmann equations

Physics of Fluids 29, 046103 (2017); 10.1063/1.4981227

Effect of viscosity on droplet-droplet collisional interaction

Physics of Fluids 29, 067102 (2017); 10.1063/1.4984081

Effect of Carreau-Yasuda rheological parameters on subcritical Lapwood convection in horizontal porous cavity saturated by shear-thinning fluid

Physics of Fluids 29, 063101 (2017); 10.1063/1.4986794

Droplet impact onto a solid sphere: Effect of wettability and impact velocity

Physics of Fluids 29, 062111 (2017); 10.1063/1.4990088

Droplets passing through a soap film

Physics of Fluids 29, 062110 (2017); 10.1063/1.4986798

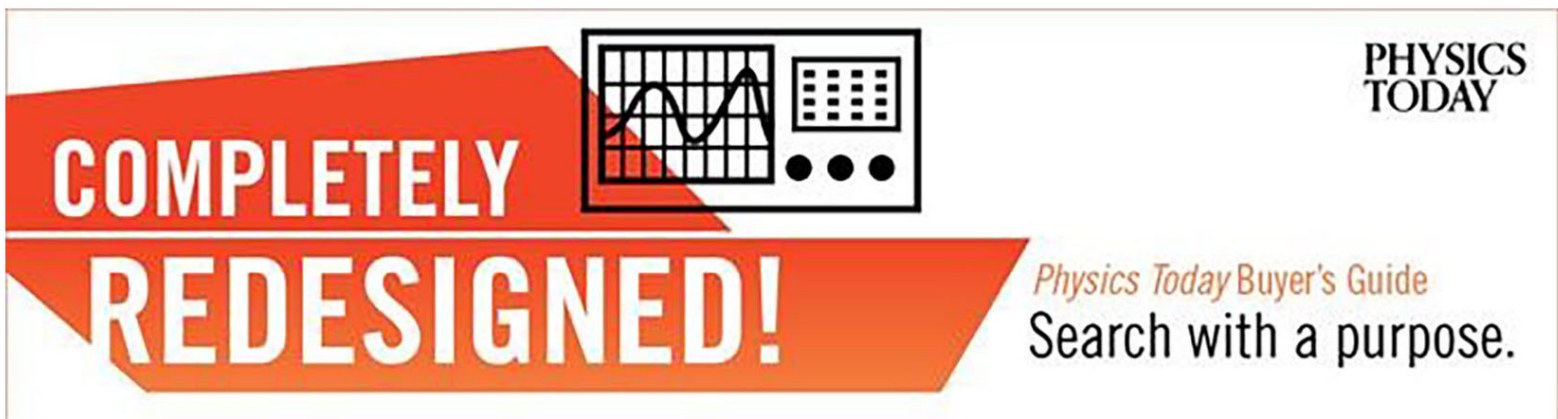




\title{
Effect of initial densities in the lattice Boltzmann model for non-ideal fluid with curved interface
}

\author{
Jiaming Gong ${ }^{1,2, a)}$ and Nobuyuki Oshima ${ }^{2}$ \\ ${ }^{1}$ Institute of Industrial Science, The University of Tokyo, Tokyo 153-8505, Japan \\ ${ }^{2}$ Department of Mechanical and Space Engineering, Hokkaido University, Sapporo 060-6868, Japan
}

(Received 4 March 2017; accepted 9 June 2017; published online 28 June 2017)

\begin{abstract}
The effect of initial densities in a free energy based two-phase-flow lattice Boltzmann method for non-ideal fluids with a curved interface was investigated in the present work. To investigate this effect, the initial densities in the liquid and gas phases coming from the saturation points and the equilibrium state were adopted in the simulation of a static droplet in an open and a closed system. For the purpose of simplicity and easier comparison, the closed system is fabricated by the implementation of the periodic boundary condition at the inlet and outlet of a gas channel, and the open system is fabricated by the implementation of a constant flux boundary condition at the inlet and a free-out boundary condition at the outlet of the same gas channel. By comparing the simulation results from the two types of initial densities in the open and closed systems, it is proven that the commonly used saturation initial densities setting is the reason for droplet mass and volume variation which occurred in the simulation, particularly in the open system with a constant flux boundary condition. Such problems are believed to come from the curvature effect of the surface tension and can be greatly reduced by adopting the initial densities in the two phases from equilibrium state. Published by AIP Publishing. [http://dx.doi.org/10.1063/1.4989638]
\end{abstract}

\section{INTRODUCTION}

Multiphase flows, especially the one-component-twophase flows, such as the liquid/gas two-phase flow systems of refrigerant and water, occur in many natural and industrial instances. In such systems, the physics occurring in the mesoscopic interface comes from the microscopic intermolecular interactions of the two phases or components and usually dominates the macroscopic performances of the whole twophase flow system. Due to such a broad range of spatial and time scales involved in the interfacial dynamics, it is difficult for traditional techniques to appropriately simulate the macroscopic two-phase phenomena in these systems. ${ }^{1,2}$ The microscopic techniques, for example, the molecular dynamics (MD) which basically make use of the real microscopic physics among the interacting molecules simulate the interface dynamics by tracking the movements of each molecule, ${ }^{3}$ are able to accurately simulate microscopic multiphase and multicomponent phenomena. However, these methods require large computational resources, so that they are impractical for macroscopic phenomena. On the other hand, the macroscopic continuum theory based methods, such as the $\mathrm{VOF}^{4,5}$ the $\mathrm{LS},{ }^{6,7}$ and the CIP, ${ }^{8,9}$ are able to simulate the macroscopic multiphase and multi-component flows, but all of them have difficulty in capturing the details of the physics occurring in the interface, so they are found to be deficient in dynamic problems.

\footnotetext{
a) Author to whom correspondence should be addressed: gjmhit@ hotmail.com.
}

For the past twenty five years, the academic society has been making efforts to construct mescoscopic theories based numerical methods to connect the microscopic and macroscopic modeling of the interface physics, among which the lattice Boltzmann method (LBM) based models have been found to be effective in dealing with the multiphase and multi-component flow problems. ${ }^{1,10-14}$ Due to the kinetic nature, the mesoscopic LBM model can directly incorporate the microscopic descriptions of the physics occurring in the interface which are the origins of phase segregation and surface tension; meanwhile, the predicted macroscopic quantities satisfy the desired macroscopic Navier-Stokes equations set well. Since 1990s, there have been several popular multiphase/multi-component LBM models presented in the literatures. The first one is the color-gradient model developed by Gunstensen et al. ${ }^{15}$ which is based on a two-component lattice gas model proposed by Rothman and Keller. ${ }^{16}$ The second type is the pseudo-potential model proposed by Shan and Chen, ${ }^{17,18}$ in which the microscopic interaction potentials between surrounding particles are introduced. The third one is the free energy model developed by Swift et al., ${ }^{19,20}$ where the thermodynamic equilibrium pressure tensor for nonideal fluid is directly combined with the collision operator. He et $a .^{21}$ also proposed a LBM model for a multiphase flow based on the continuous discrete Boltzmann equation, and this type of model is also referred to as the kinetic theory based model. Because both the free energy model and the kinetic theory based model are tightly connected with the Cahn-Hilliard equation of the phase filed theory that describes the thermodynamics of a non-uniform fluid system, ${ }^{22}$ sometimes the kinetic theory based model is also categorized as a form of the free energy based model ${ }^{23}$ although the origins of the two 
models are different. The free energy based LBM models satisfy the thermodynamic consistency; also, it is believed that the spurious velocity in this type of models decreases greatly compared with the other models. Recently, the free energy based LBM models have been becoming popular in real applications related with two-phase simulations in micro-channels, like the droplet removal mechanism in the polymer electrolyte fuel cell (PEFC). ${ }^{24-26}$

According to the scale analysis or the so-called ChapmanEnskog expansion, the LBM models, no matter the basic LBM for single phase flow or the LBM models for multiphase and multi-component flows, can be recovered to a macroscopic incompressible Navier Stokes equation with a second order accuracy. ${ }^{11-13,27}$ In these incompressible flow simulations with LBM models, people usually ignore the slight compressibility of the LBM models and treat the densities in the flow as constant. In the LBM models for non-ideal fluids (also be called as the one-component-two-phase flow LBM models), no matter the pseudo-potential model, the free energy model, or the kinetic theory based model, people usually use the densities in the two phases across a flat interface as the initial densities in their simulations, ${ }^{24,25,28-33}$ except for the simulations of phase segregation in which the initial density is set to be in the middle range of the two phases. ${ }^{13}$ The initial densities in the liquid and gas phases of a two-phase flow system with a flat interface are also the saturation densities in the two phases. According to the phase diagram of a two-phase flow system, no matter it is described by the van der Waals equation of state (EOS), the Carnahan-Starling EOS, or the SC $\mathrm{EOS}^{34}$ and so on, the thermodynamic pressures at the saturation points of the two phases are the same, so there is no pressure difference across the interface to keep the force balance in the two phases of a flat interface system. This initial density setting method is easy and reasonable; however, the flow patterns encountered in practical simulations are not limited in flat interfaces; in fact, most scenarios encountered in these situations involved curved interfaces. Due to the interface tension effect, there should be a pressure difference across the interface to keep force balance in the steady or equilibrium state. Correspondingly, the densities in the two phases across the curved interface should not be the saturation densities or the densities in the two phases across the flat interface. In these cases, starting simulations with the densities at the saturation points should introduce some error or effect to the two-phase flow system; however, no one has ever noticed this problem.

On the one hand, the most representative flow pattern in a two-phase flow system with curved interfaces is the droplet dynamics, which is of great interest in both scientific and engineering. On the other hand, the theoretical solutions of a static droplet are easy to acquire. So in this paper, investigations are going to be implemented by simulating a static droplet in the quiescent gas environment. Besides, since in real industrious process a droplet is manipulated in either an open or a closed system, to investigate the effect of the initial densities in the two typical system environments and for the purposes of simplicity and easier comparison, the static droplet is going to be simulated in a gas channel with the inlet and outlet to be connected by the periodic boundary condition to fabricate a closed system and with the inlet and outlet to be separately set with constant flux and free-out boundary conditions to fabricate an open system. The initial densities in the gas and liquid phases adopted in the simulations will be the densities at the saturation points and the equilibrium state.

The rest of this paper is organized as Secs. II-IV. In Sec. II, the free energy based one-component-two-phase LBM model which is proposed by Inamuro et al. ${ }^{32}$ is briefly reviewed; then the general boundary conditions and their corresponding schemes for the closed and open systems are described. In Sec. III, first, the initial condition and parameters adopted in the simulations are given; then, numerical results and analyses with different initial densities in the two types of system are presented. Finally, in Sec. IV, a summary is made to get the general conclusion.

\section{NUMERICAL MODEL}

In this section, the free energy based LBM model for onecomponent-two-phase flows with a large density ratio, which is originally proposed by Inamuro et al., ${ }^{32}$ is briefly reviewed. Then, the detailed boundary conditions used in a gas channel to fabricate the closed system and the open system are introduced, respectively.

\section{A. The free energy based LBM model for non-ideal fluid}

Inamuro et al. proposed a free energy based LBM model for non-ideal fluid, which consists of two distribution functions. In this model, the non-dimensional variables are defined by a characteristic length, $\hat{L}$, a characteristic particle velocity, $\hat{c}$, and a characteristic time scale, $\hat{t}_{0}=\hat{L} / \hat{U}$, where $\hat{U}$ is the characteristic flow speed. The real physical parameters are expressed by a circumflex and the "non-dimensional" ones are expressed without circumflex. A reference density, $\hat{\rho}_{0}$, and a reference order parameter, $\hat{\phi}_{0}$, are also used to make dimensionless two phase density $\rho=\hat{\rho} / \hat{\rho}_{0}$ and order parameter $\phi=\hat{\phi} / \hat{\phi}_{0}$. The two particle velocity distribution functions are represented by $f_{i}$ and $g_{i}$. The function $f_{i}$ is used for the calculation of an order parameter $\phi$ which distinguishes two phases: $\phi<\phi_{G}$ corresponds to the gas phase, $\phi_{G} \leq \phi \leq \phi_{L}$ corresponds to the interface, and $\phi>\phi_{L}$ corresponds to the liquid phase. The function $g_{i}$ is used to calculate the predicted velocity of the two-phase fluid without a pressure gradient. The evolution functions of the two particle functions $f_{i}$ and $g_{i}$ are described by the following equation:

$$
\begin{aligned}
f_{i}\left(\mathbf{x}+\mathbf{c}_{i} \Delta t, t+\Delta t\right)= & f_{i}(\mathbf{x}, t)-\frac{1}{\tau_{f}} \cdot\left(f_{i}(\mathbf{x}, t)-f_{i}^{e q}(\mathbf{x}, t)\right), \quad(1) \\
g_{i}\left(\mathbf{x}+\mathbf{c}_{i} \Delta t, t+\Delta t\right)= & g_{i}(\mathbf{x}, t)-\frac{1}{\tau g}\left(g_{i}(\mathbf{x}, t)-g_{i}^{e q}(\mathbf{x}, t)\right) \\
& +\frac{3 E_{i} c_{i \alpha}}{\rho}\left[\frac{\partial}{\partial x_{\beta}}\left\{\mu\left(\frac{\partial u_{\beta}}{\partial x_{\alpha}}+\frac{\partial u_{\alpha}}{\partial x_{\beta}}\right)\right\}\right] \Delta x .
\end{aligned}
$$

Here, $f_{i}^{e q}$ and $g_{i}^{e q}$ are the equilibrium distribution functions, $\tau_{f}$ and $\tau g$ are the dimensionless single relaxation times, $E \mathrm{i}$ are 
the associated weight coefficients presented below, $\Delta x$ is the spacing of the cubic lattice, $\Delta t$ is the time step during which the particles travel across the distance of a lattice spacing, $\rho$ is the density, $\mu$ is the viscosity, and $\boldsymbol{u}$ is the current velocity. The subscripts $\alpha$ and $\beta(=x, y)$ represent Cartesian coordinates, and the summation convection is used. The third term on the right side of Eq. (2) represents the effects of viscous stress. The gravitation effect is not included in the present study.

For the two-dimensional 9 velocities model (2DQ9 model), the velocities of particle ensembles which depend on the lattice velocity directions as shown in Fig. 1 are listed as follows:

$$
\begin{aligned}
{\left[\boldsymbol{c}_{1}, \boldsymbol{c}_{2}, \boldsymbol{c}_{3}, \boldsymbol{c}_{4}, \boldsymbol{c}_{5}, \boldsymbol{c}_{6}, \boldsymbol{c}_{7}, \boldsymbol{c}_{8}, \boldsymbol{c}_{9}\right] } \\
\quad=\left[\begin{array}{rrrrrrrrr}
0 & 1 & 0 & -1 & 0 & 1 & -1 & -1 & 1 \\
0 & 0 & 1 & 0 & -1 & 1 & 1 & -1 & -1
\end{array}\right] .
\end{aligned}
$$

The order parameter $\phi$ distinguishing two phases and the predicted velocity $\boldsymbol{u}^{*}$ of the two-phase fluid with a large density ratio are defined in terms of the two particle velocity distribution functions. Here $N$ is the velocity direction number, and for the $2 \mathrm{D}$ model $N$ is 9 ,

$$
\begin{gathered}
\phi=\sum_{i=1}^{N} f_{i}, \\
\boldsymbol{u}^{*}=\sum_{i=1}^{N} \boldsymbol{c}_{i} g_{i} .
\end{gathered}
$$

The equilibrium distribution functions $f_{i}^{e q}$ and $g_{i}^{e q}$ in Eqs. (1) and (2) are given as follows:

$$
\begin{aligned}
f_{i}^{e q}= & H_{i} \phi+F_{i}\left[p_{0}-\kappa_{f} \phi \frac{\partial^{2} \phi}{\partial x_{\alpha}^{2}}-\frac{\kappa_{f}}{6}\left(\frac{\partial \phi}{\partial x_{\alpha}}\right)^{2}\right] \\
& +3 E_{i} \phi c_{i \alpha} u_{\alpha}+E_{i} \kappa_{f} G_{\alpha \beta}(\phi) c_{i \alpha} c_{i \beta} \\
g_{i}^{e q}= & E_{i}\left[1+3 c_{i \alpha} u_{\alpha}-\frac{3}{2} u_{\alpha} u_{\alpha}+\frac{9}{2} c_{i \alpha} c_{i \beta} u_{\alpha} u_{\beta}+\frac{3}{2}\left(\tau g-\frac{1}{2}\right)\right. \\
\times & \left.\Delta x\left(\frac{\partial u_{\beta}}{\partial x_{\alpha}}+\frac{\partial u_{\alpha}}{\partial x_{\beta}}\right) c_{i \alpha} c_{i \beta}\right]+E_{i} \frac{\kappa_{g}}{\rho} G_{\alpha \beta}(\rho) c_{i \alpha} c_{i \beta} \\
- & \frac{1}{2} F_{i} \frac{\kappa_{g}}{\rho}|\nabla \rho|^{2},
\end{aligned}
$$

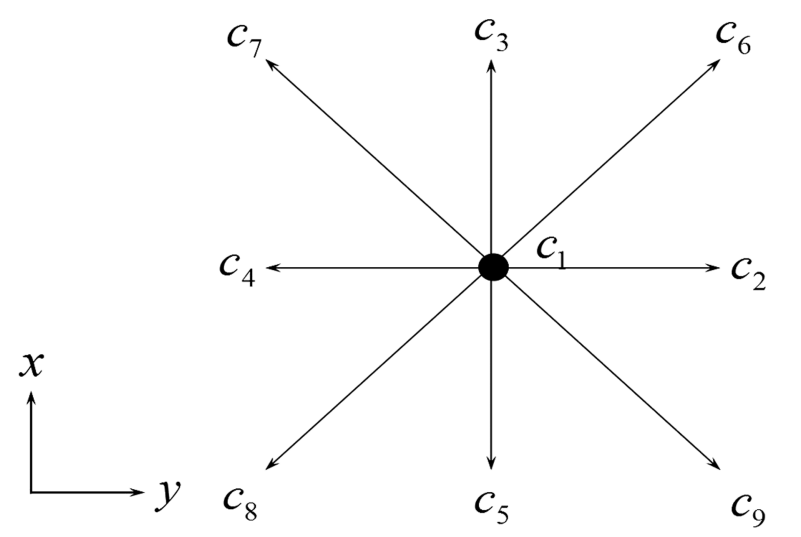

FIG. 1. Lattice structure of two-dimensional 9 velocities model (2DQ9 model). where

$$
\begin{gathered}
E_{1}=\frac{4}{9}, E_{2}=E_{3}=E_{4}=E_{5}=\frac{1}{9}, E_{6}=E_{7}=E_{8}=E_{9}=\frac{1}{36} \\
H_{1}=1, H_{2}=H_{3}=\cdots=H_{9}=0, F_{1}=-\frac{5}{3} \\
F_{i}=3 E_{i}(i=2,3, \ldots, 9),
\end{gathered}
$$

and

$$
G_{\alpha \beta}(\lambda)=\frac{9}{2} \frac{\partial \lambda}{\partial x_{\alpha}} \frac{\partial \lambda}{\partial x_{\beta}}-\frac{9}{4} \frac{\partial \lambda}{\partial x_{\gamma}} \frac{\partial \lambda}{\partial x_{\gamma}} \delta_{\alpha \beta},
$$

where $\alpha, \beta$, and $\gamma$ are the Cartesian coordinates following the summation convection and $\delta_{\alpha \beta}$ is the Kronecker delta. $\kappa_{f}$ is a constant parameter that determines the interface width between the liquid and the gas phases and $\kappa_{g}$ is a constant parameter that determines the strength of the surface tension in the large density ratio system. Additionally, $\lambda$ in Eq. (8) is the free parameter used to represent $\phi$ and $\rho$ in Eqs. (6) and (7). Correspondingly, $p_{0}$ is the pressure of the van der Waals fluid described by $\phi$, where the equation of state (EOS) is defined by

$$
p_{0}=\phi \frac{\partial \psi}{\partial \phi}-\psi=\phi T \frac{1}{1-b \phi}-a \phi^{2}
$$

with

$$
\psi(\phi, T)=\phi T \ln \left(\frac{1}{1-b \phi}\right)-b \phi
$$

Here $\psi$ is the bulk free-energy density, and $a, b$, and $T$ are the free parameters determining the maximum and minimum values of the order parameter $\phi$.

As the two-phase system described by the order parameter $\phi$ is limited to small density ratio, the cutoff and magnification around the interface region are adopted to realize large density ratio around 1000:1 in the following way:

$\rho=\left\{\begin{array}{cl}\rho_{G}, & \phi \leq \phi_{G} \\ \frac{\Delta \rho}{2}\left[\sin \left(\frac{\phi-\bar{\phi}}{\Delta \phi} \pi\right)+1\right]+\rho_{G}, & \phi_{G}<\phi<\phi_{L}, \\ \rho_{L}, & \phi \geq \phi_{L}\end{array}\right.$

where $\Delta \rho=\rho_{L}-\rho_{G}, \bar{\phi}=\left(\phi_{L}+\phi_{G}\right) / 2, \Delta \phi=\phi_{L}-\phi_{G}$.

Because the predicted velocity $\boldsymbol{u}^{*}$ calculated by Eq. (5) does not satisfy the continuity equation $\left(\nabla \cdot \boldsymbol{u}^{*}=0\right)$, the correction of $\boldsymbol{u}^{*}$ is needed. The current velocity $\boldsymbol{u}$ which satisfies the continuity equation can be obtained by the fractional step method with the following equations:

$$
\begin{aligned}
& S h \frac{\boldsymbol{u}-\boldsymbol{u}^{*}}{\Delta t}=-\frac{\nabla p}{\rho}, \\
& \nabla \cdot\left(\frac{\nabla p}{\rho}\right)=S h \frac{\nabla \cdot \boldsymbol{u}^{*}}{\Delta t} .
\end{aligned}
$$

Here, $S h=U / c$ is the Strouhal number and $p$ is the pressure of the two-phase fluid with a large density ratio that is described by $\rho$. It is noted that $\Delta t=S h \Delta x$, and in real application, after the non-dimensionalization, $\Delta x=1$ and $\Delta t=1$ are adopted to simplify Eqs. (12) and (13). For a more detailed description about this model, please refer to the literatures. $^{24,32,35,36}$ 


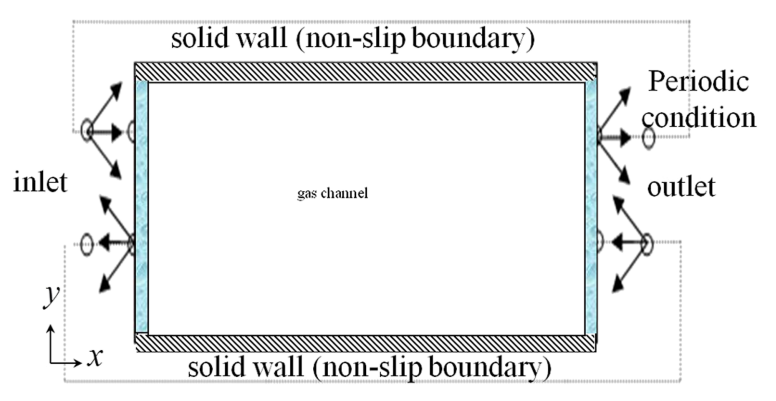

FIG. 2. Boundary conditions adopted in a gas channel to fabricate a closed system.

\section{B. Boundary conditions for a closed and open gas channel system}

The sketch map of the gas channel adopted in the work is illustrated in Figs. 2 and 3. The vertical inlet and outlet are set on the left and right hand side of the gas channel, respectively. As shown in Figs. 2 and 4, when the periodic boundary condition is implemented at the inlet and outlet along the $x$ direction, the computational domain becomes a circular loop, and the gas channel becomes a closed system. For an open system or a gas channel with open boundaries, the most common scenario is the constant flux inlet (or the so-called velocity inlet) and the free-out outlet (or the so-called zero-gradient outlet), ${ }^{12,37}$ so such boundary conditions are adopted in this work to verify the effect of initial densities in the open system. For simplicity, the constant flux inlet boundary condition is realized by the equilibrium boundary scheme, ${ }^{12,13}$ the freeout outlet boundary condition is realized by the fully developed boundary scheme, ${ }^{12,13}$ and the non-slip boundary condition at

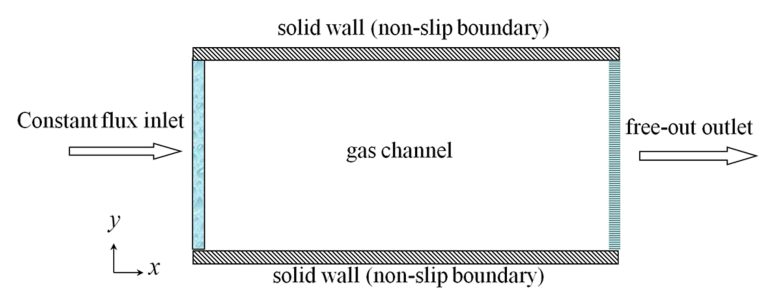

FIG. 3. Boundary conditions adopted in a gas channel to fabricate an open system.

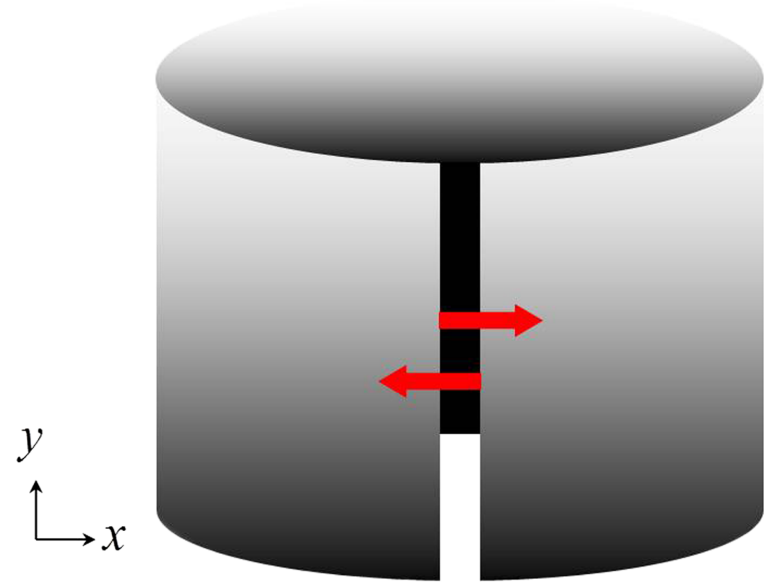

FIG. 4. Schematic of the periodic boundary condition along the $x$ direction. the solid wall of gas channel is realized by the general mid-grid bounce-back scheme. ${ }^{12,13,38}$

\section{NUMERICAL RESULTS}

\section{A. Initial conditions and parameters}

To investigate the effect of initial densities in the free energy based two-phase flow LBM model for non-ideal fluids with curved interfaces, a static droplet was assigned in the center of a gas channel with a stationary gas phase around it, as shown in Fig. 5. In this figure, a water droplet with a radius of $12 d x(R=12 d x)$ is placed in the center of the calculation domain; here, the red region is the liquid water and the blue region is its vapor or the gas phase. The lengths of the domain in the $x$ and $y$ directions are $\mathrm{LX}=91 d x$ and $\mathrm{LY}=61 d x$. The two-phase intermolecular interaction parameters in Eq. (9) are set to be $a=1, b=6.7, T=3.5 \times 10^{-2}$; correspondingly, the saturation order parameters in the gas and liquid phases are $\phi_{G 0}=1.134 \times 10^{-2}$ and $\phi_{L 0}=9.714 \times 10^{-2}$, respectively, which are also the saturation densities of the two-phase system described by the order parameter $\phi$. Figure 6 shows the phase diagram described by Eq. (9) under the above parameters. In the simulation, $\phi_{G}=1.15 \times 10^{-2}$ and $\phi_{L}=9.20 \times 10^{-2}$ are used to distinguish the two phases for the two-phase flow system with a large density ratio, and in this paper the two-phase flow system of liquid water and its vapor at $23{ }^{\circ} \mathrm{C}$ is chosen to be the large density ratio system which is described by the density $\rho$, as shown in Eqs. (2), (7), and (11). The densities of liquid water and its vapor are $\rho_{L}=996.62 \mathrm{~kg} / \mathrm{m}^{3}$ and $\rho_{G}$ $=1.1763 \mathrm{~kg} / \mathrm{m}^{3}$; the viscosities are $\mu_{L}=8.544 \times 10^{-4} \mathrm{~Pa}$ and $\mu_{G}=1.862 \times 10^{-5} \mathrm{~Pa} \mathrm{~s}$; the surface tension is $\sigma=0.07288 \mathrm{~N} \mathrm{~s}$; the characteristic particle speed $c$ is $200 \mathrm{~m} / \mathrm{s}$. The surface tension related constants in Eqs. (6) and (7) are $\kappa_{f}=0.5$ and $\kappa_{g}=4.916 \times 10^{-8}$, respectively. A square lattice $(d x=d y$ $=1.33 \times 10^{-4} \mathrm{~m}$ ) is used in the LBM evolution, and the $\tau_{f}$ and $\tau_{g}$ are 1 in Eqs. (1) and (2). Besides, for simplicity and easier comparison, the inlet velocity in the case of an open system is set to be uniformly $0 \mathrm{~m} / \mathrm{s}$, so that a static droplet in the steady state is expected in both the closed and open systems.

Because the density profile ( $\rho$ profile) of the two-phase system with a large density ratio is determined by the density profile ( $\phi$ profile) of the two-phase system with a small density ratio, as shown in Eq. (11), and after the cutoff and

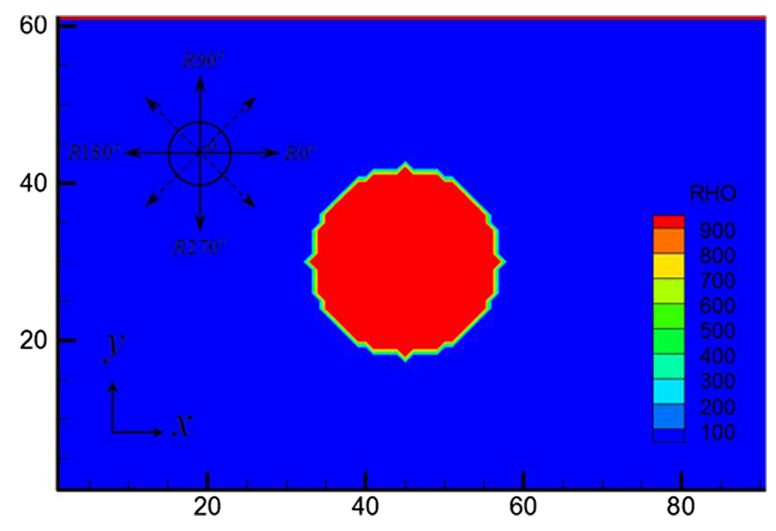

FIG. 5. Initial assignment of the two phases. 


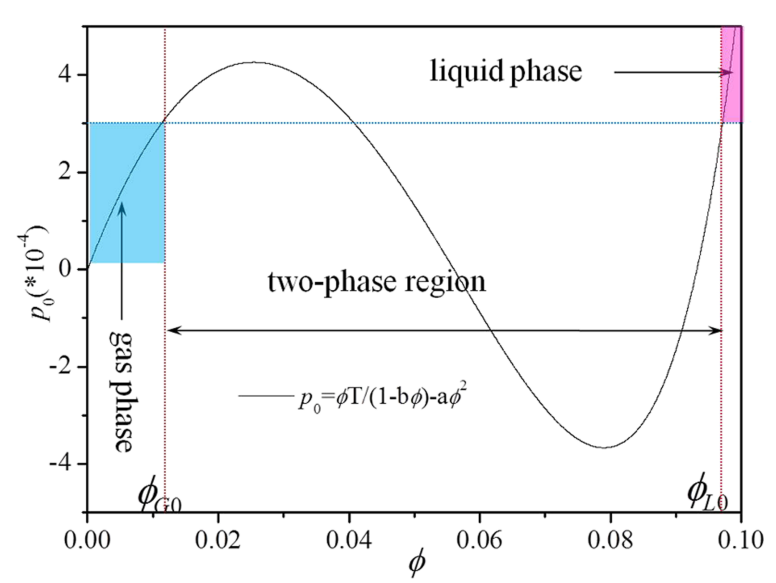

FIG. 6. The pressure-density isotherm phase diagram at $a=1, b=6.7$, and $T$ $=0.035$ [Eq. (9)].

magnification process, the $\rho$ profile becomes incompressible. So the effect of the initial densities in the present model eventually comes from the initial densities of the small density ratio system, namely, the initial values of the order parameter $\phi$. The initial order parameter $\phi$ 's distribution at the liquid/gas interface is un-physical as it changes suddenly and sharply from $\phi_{L 0}$ to $\phi_{G 0}$ in one lattice point (from the liquid phase to the gas phase), correspondingly the density $\rho$ changes from almost 1000 to 1 (as the black line shown in Fig. 7) and the initial droplet figure is not smooth (as shown in Fig. 5). So the simulation process is expected to first pass through an unsteady stage which forms a liquid/gas interface with an appropriate density profile inside it and a smooth figure.

\section{B. The effect of initial densities in the closed gas channel}

When a static droplet is simulated in a closed gas channel, it is found that even if the appropriate density (for the small density ratio system, $\phi$; for the large density ratio system, $\rho$ ) profile of the interface and the smooth circular figure of the droplet have been formed, the unsteady stage would continue for a much longer time, and during this process, the volume and mass of the droplet would continuously increase until the two-phase flow system reaches the equilibrium state.

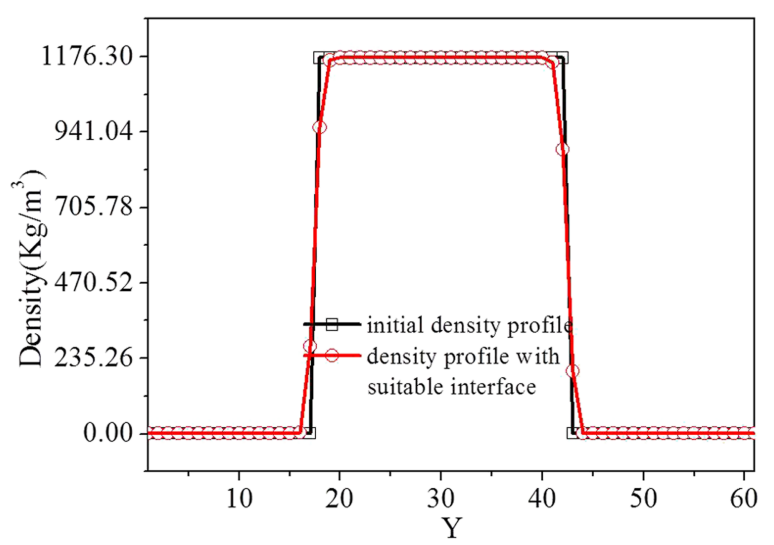

FIG. 7. Density profiles of liquid water/gas system across the center of the calculation domain.

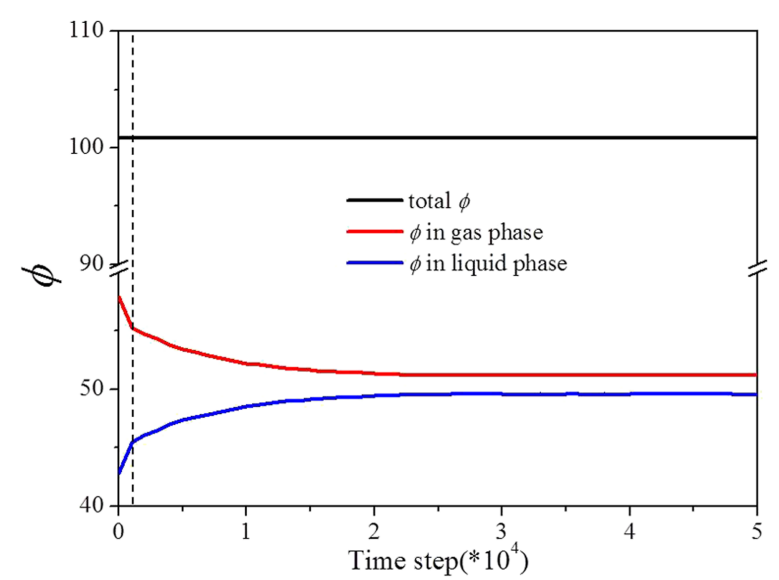

FIG. 8. Evolution histories of the mass in the small density ratio system in the closed gas channel with initial $\phi$ in the two phases from the saturation points.

Figure 8 shows the evolution histories of the total $\phi$, the $\phi$ in the gas phase, and the $\phi$ in the liquid phase in the whole domain. From Fig. 8, we can find that the total $\phi$ in the whole domain is always constant, and the $\phi$ in the gas phase is gradually decreasing in the unsteady stage; meanwhile, the $\phi$ in the liquid phase is continuously increasing until the simulation reaches the steady stage or the equilibrium state. The constant total $\phi$ in the whole domain is attributed to the implementation of the periodic boundary condition at the inlet and outlet of the gas channel, as such treatment makes the gas channel a closed system and no mass exchange with outside would happen. The increased $\phi$ in the liquid phase is believed to come from the $\phi$ decrease in the gas phase in the unsteady state and it will lead to the mass increase and volume expansion of the water droplet. Figure 9 shows the corresponding evolution histories of the total mass, the mass in the gas phase, and the mass in the liquid phase of the whole domain in the large density ratio system. The basically constant (indeed slightly decreasing) gas mass in the whole domain is believed to come from the cutoff and magnification process to get the $\rho$ profile from $\phi$ profile, as no matter how much $\phi$ decreased in a single lattice in the gas phase, only if its initial value is smaller than $\phi_{G}$, its corresponding density in the large density ratio system would

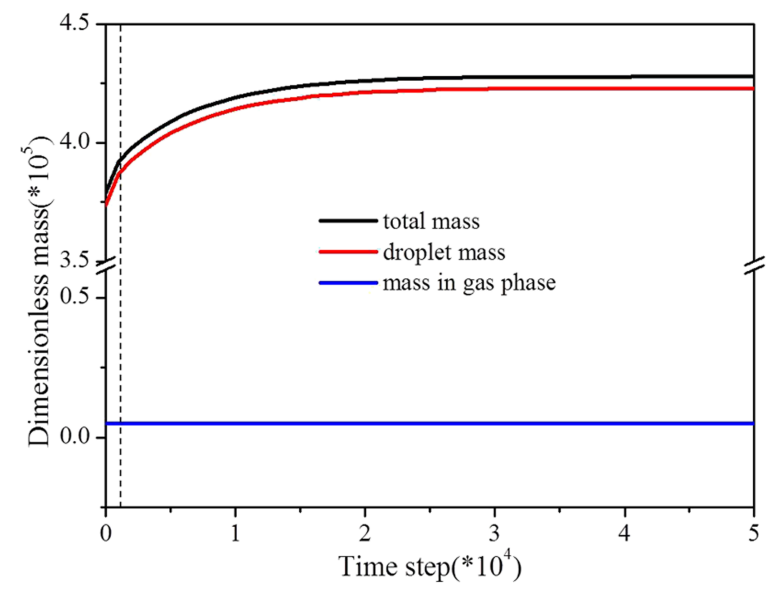

FIG. 9. Evolution histories of the mass in the large density ratio system in the closed gas channel with initial $\phi$ in the two phases from the saturation points. 


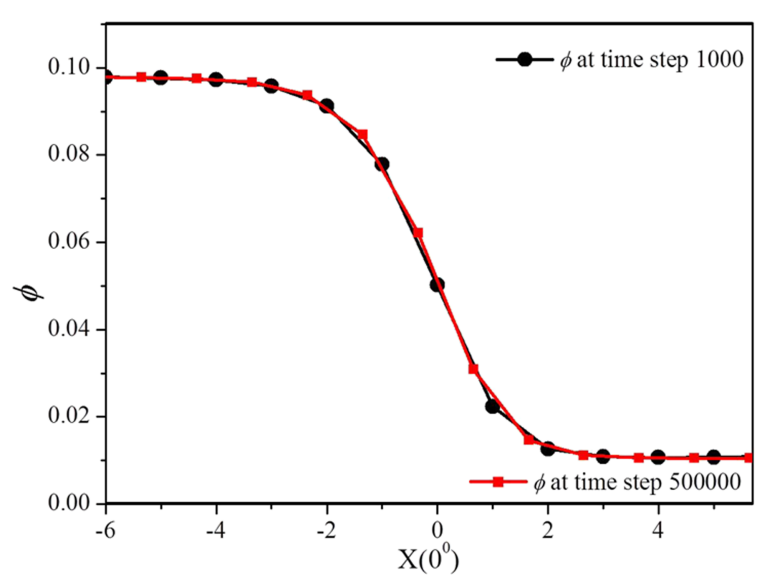

FIG. 10. Interface profile of $\phi$ at certain time steps of the beginning and steady stage in the simulation.

constantly be $\rho_{G}$. The constant mass in the gas phase together with the increasing mass in the liquid phase makes the total mass in the whole domain with the same variation tendency with the liquid mass. The black dotted line in Figs. 8 and 9 indicates the time step when the interface profile of $\phi$ or $\rho$ has already converged. Figure 10 shows the interface profiles of the order parameter $\phi$ at a time step of 1000 (the time step shown by the black dotted line in Figs. 8 and 9) and a time step of 500000 (in the steady stage) of the simulation, and in this figure the interface center is assigned as the origin of the coordinate, from this figure it is found that there is no obvious difference between two profiles at the two time steps and this proves that the interface profile of $\phi$ or $\rho$ has already converged at the very beginning of the unsteady stage. Figure 11 illustrates the corresponding density distributions of the two-phase system with a large density ratio at the two time steps; the black dotted circle shows the theoretical volume of the droplet at the equilibrium state. Here, as the spatial distribution of $\rho$ and $\phi$ is basically the same and to be consistent with Fig. 5, in Fig. 11 we only show the density distribution of $\rho$. From Fig. 11(a), we can find that the smooth droplet figure has already formed at the very beginning stage of the unsteady stage and can compare Figs. 11(a) and 11(b); it is found that during the evolution process from the unsteady stage to the steady stage, the main difference of the two figures is the symmetrical volume expansion of the droplet. To quantify the volume expansion process, Fig. 12 which shows the evolution histories of droplet radii along different directions (as illustrated in the inset) is plotted. Due to the symmetry observed in the volume expansion process, only the radii along the $0^{\circ}$ and $45^{\circ}$ directions are expressed in this figure. The average length of the two radii is used to express the volume expansion degree of the droplet. As the radius or volume expansion only occurred in the unsteady stage, to make a clearer observation in the whole process, the logarithmic coordinate is adopted in Fig. 12(b). However, such a coordinate cannot truly show the volume variation history at the beginning of the unsteady stage, so Fig. 12(a) with the linear coordinate is adopted for supplementation. The radius lengths along the two directions are measured from the droplet center to the top lattice points in the liquid phase at the interface, due to the initial zigzag droplet shape, as shown in Fig. 5, the initial radius lengths along the two directions are larger than the averaged initial radius length. When simulation starts, these overshot tops would be removed by the interface tension effect, leading to a sharp radius length shrinking at the tops of the zigzag interface, as shown in the very beginning stage of Fig. 12(a). But the actually averaged radius length or volume in this short process would basically remain the same.

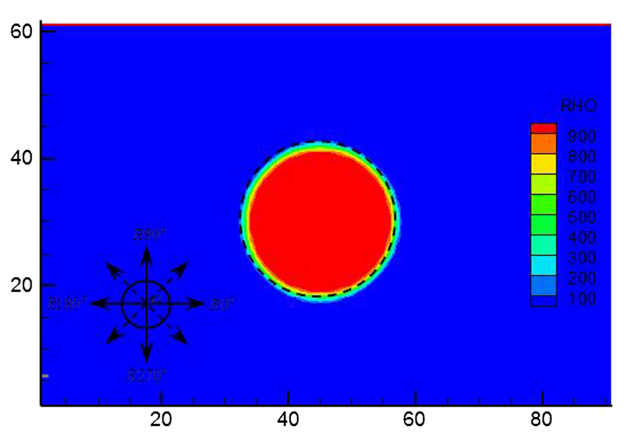

(a)

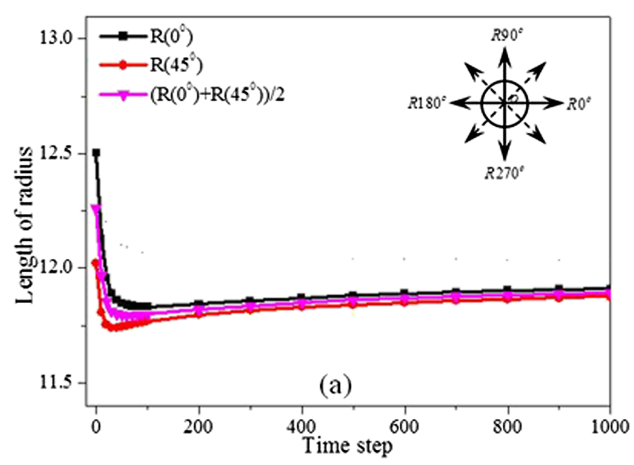

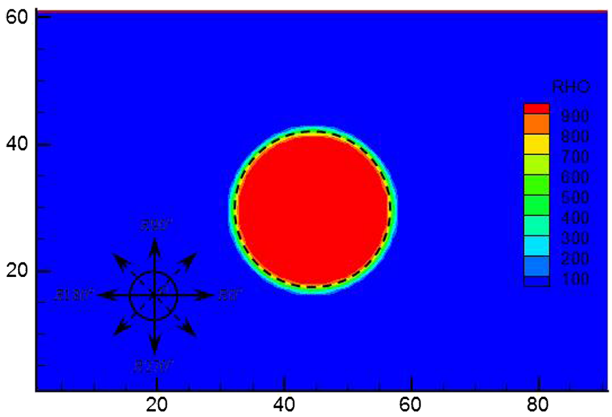

(b)

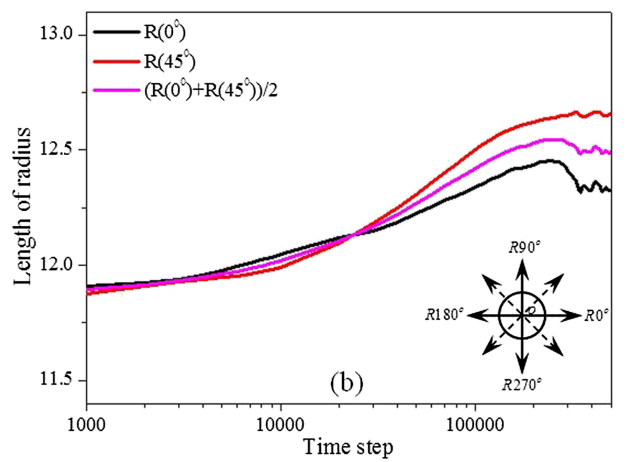

FIG. 11. Density distributions of the two-phase flow system at different stages in a closed gas channel with initial $\phi$ in the two phases from the saturation points. (a) Time step $=1000$. (b) Time step $=500000$.
FIG. 12. Evolution histories of droplet radii and volume in a closed gas channel with initial $\phi$ in the two phases from the saturation points. 


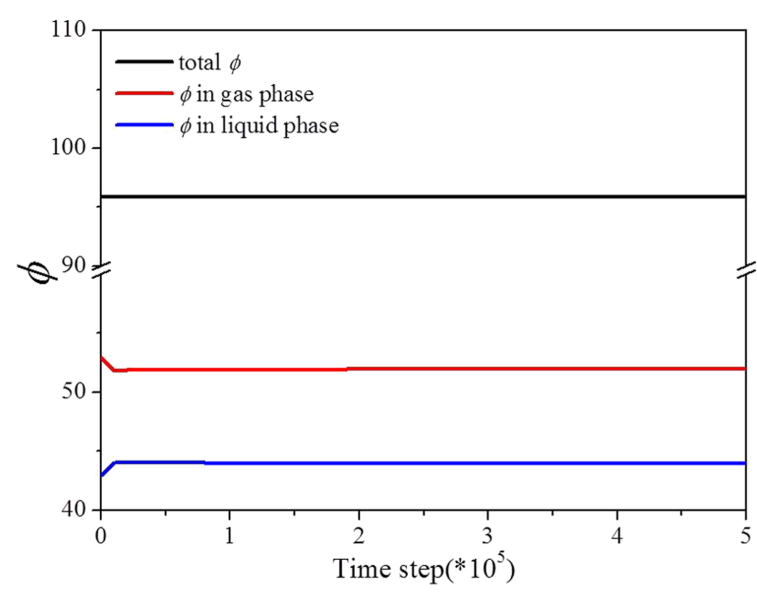

FIG. 13. Evolution histories of the mass in the small density ratio system in the closed gas channel with initial $\phi$ in the two phases from the equilibrium state.

The slight radius length and volume variation shown in the steady stage region of Fig. 12(b) is believed to come from the movement of droplet center, owing to the existence of numerical error, for example, the artificial velocity field. Indeed, the radii and volume of the droplet remain the same in the steady stage.

We believe that the mass and volume variation observed above comes from the inappropriate initial density settings in the two phases. When there is a curved interface in a twophase flow system, the saturation densities or the densities of a two-phase flow system with a flat interface should not be used as the initial densities, instead, the densities in the equilibrium state should be used as the initial densities. To test our idea, the averaged densities (order parameter, $\phi$ ) in the gas and liquid phases (excluding the interface region) of the two-phase flow system with small density ratio acquired in the steady stage (equilibrium state) of the above simulation are adopted as the new initial densities, with their values to be $\phi_{G 1}=1.036 \times 10^{-2}$ and $\phi_{L 1}=9.742 \times 10^{-2}$, respectively. In the new simulation with the equilibrium state densities, the initial spatial arrangement of the two phases is the same as the one in the previous case as shown in Fig. 5.

Corresponding to Figs. 8, 9, 11 and 12, Figs. 13-16 illustrate the simulation results with the initial densities in the liquid and gas phases set from the equilibrium state. As shown in Fig. 13, it is found that except for a little variation in the very beginning stage, $\phi$ in both the liquid and gas phases remain

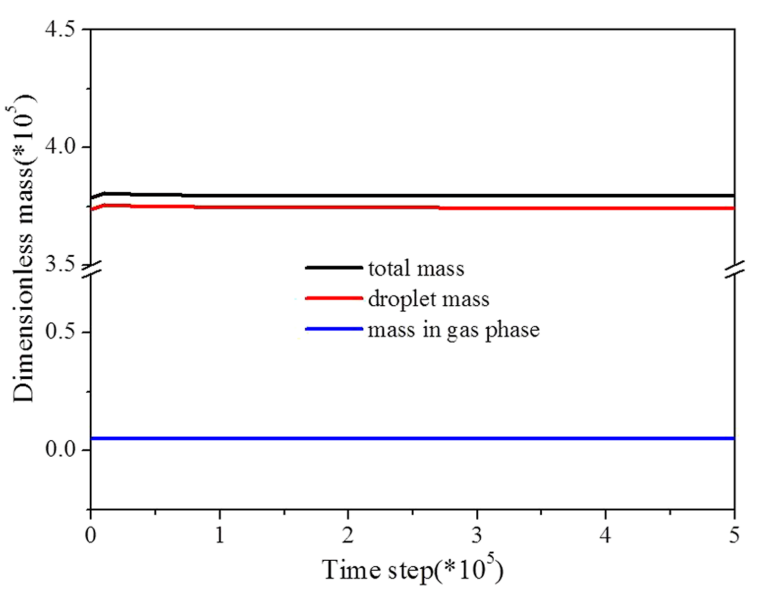

FIG. 14. Evolution histories of the mass in the large density ratio system in the closed gas channel with initial $\phi$ in the two phases from the equilibrium state.

constant in the whole simulation, and a similar tendency can be also observed in Fig. 14, which shows the mass evolution histories in the two-phase flow system described by the density $\rho$. The smaller amount of total $\phi$ and the $\phi$ in the gas phase in the whole domain in Fig. 13 compared with these in Fig. 8 is believed to come from the smaller new initial $\phi$ in the gas phase though the new initial $\phi$ in the liquid phase is slightly higher. Figures 15 and 16 show the density distributions and volume expansion history of the water droplet separately. The sharp variations of droplet volume and radius at the very beginning stage shown in Fig. 16 are believed to come from the same reason as discussed in Fig. 12. From the two figures, we can find that with the new initial densities (order parameter $\phi$ ), the volume of the droplet basically remains the same, too. These results proved our guess that the mass and volume increasing problem eventually comes from the inappropriate initial densities settings in the simulation. Naturally, the next question people may ask is how the initial densities took effect on the simulation results.

Indeed, if the interface in a two-phase flow system is flat, the choice of initial densities from the saturation points of the two phases would be a good choice. Because there would be no additional surface tension effect added to the system, as predicted by the surface tension force equation,

$$
\vec{F}_{\sigma}=\sigma \cdot C_{K} \cdot \vec{n}=\sigma \cdot \frac{1}{R} \cdot \vec{n},
$$

where $\sigma$ is the surface tension coefficient, $C_{K}$ is the curvature of an interface, $\vec{n}$ is the normal vector of the interface, and

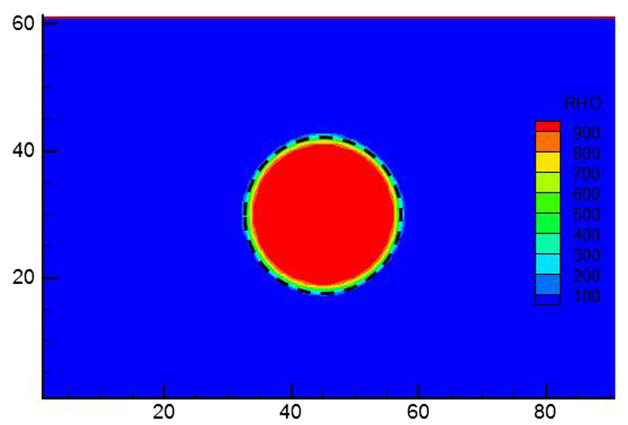

(a)

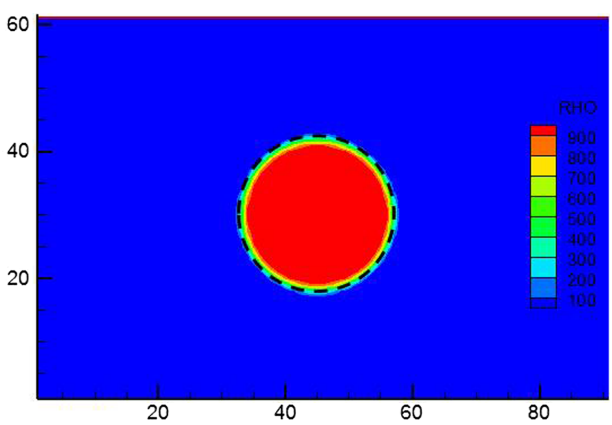

(b)
FIG. 15. Density distributions of the two-phase flow system at different stages in a closed gas channel with initial $\phi$ in the two phases from the equilibrium state. (a) Time step $=1000$. (b) Time step $=500000$. 

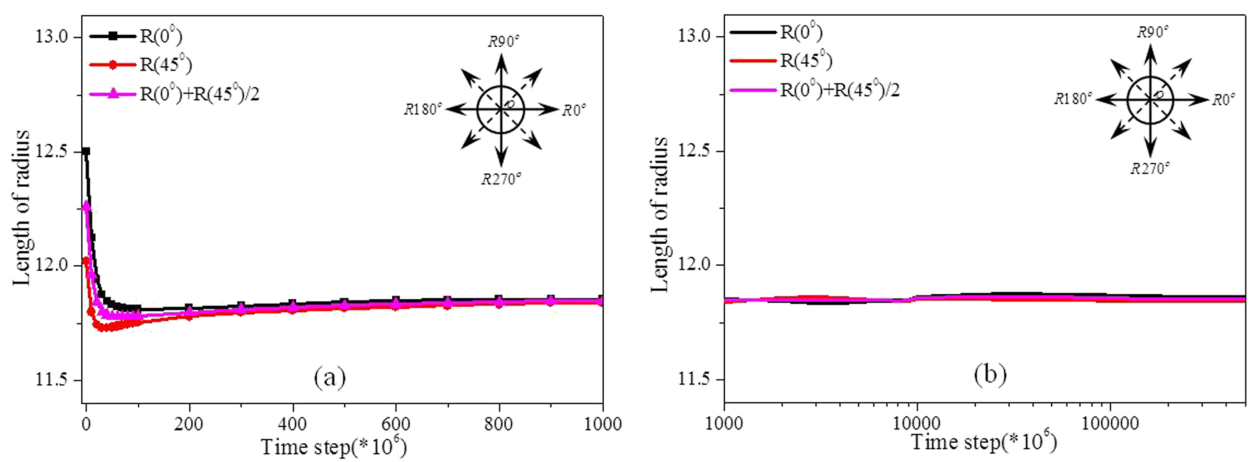

FIG. 16. Evolution histories of droplet radii and volume in a closed gas channel with initial $\phi$ in the two phases from the equilibrium state.
$R$ is the corresponding curvature radius. The curvature radius of a flat interface is $+\infty$, so there would be no effect of surface tension force; thus, there is no pressure adjustment in the two phases across the interface. The initially same pressure in the two phases given by the saturation densities [predicted by Eq. (9) and shown in Fig. 6] would keep force balance across the interface in the evolution process to the equilibrium state. However, for a curved interface, most typically, a droplet, when we start the simulation with the initial densities from the saturation points, while the initial pressure in the two phases remains the same, a strong surface tension force will be created owing to the curvature effect of the interface, according to Eq. (14). Such a strong surface tension force will compress the liquid droplet inward to increase its pressure; meanwhile, it absorbs the mass in the gas phase to the interface region to decrease the density in the gas phase, so as to decrease the pressure in the gas phase. When the created pressure difference across the interface in the two phases balances the effect of the surface tension, the two-phase flow system will reach the equilibrium state, and at this moment, the relationship between the pressure difference and the surface tension effect can be expressed by the Laplace equation for a $2 \mathrm{D}$ droplet,

$$
\Delta p=\frac{\sigma}{R} .
$$

The new pressures in the liquid and gas phases correspond to new densities in the equilibrium state: the increased pressure in the liquid phase will lead to the density increase inside the droplet, the decreased density in the gas phase will lead to the pressure decrease in the gas phase, and the absorbed mass from the gas phase into the interface region will lead to the volume expansion of the droplet. Figure 17(a) shows the initial and equilibrium order parameter profiles across the center of the droplet along the $x$ direction with the saturation densities as the initial densities in the two phases; from this figure, we can find that in the steady stage the $\phi$ in each lattice point of the gas phase is obviously lower than its initial value and the $\phi$ in liquid phase is obviously higher than its initial value. So when we start the simulation with the initial densities in the two phases from the equilibrium state, according to Eq. (9) and its diagram, a pressure difference across the droplet interface in the two phases is created in the initial stage, which is fairly enough to balance the effect of surface tension force, so the force balance in the two phases can be kept; no mass or only small mass will be absorbed into the interface region; and the volume of droplet will be kept constant. Figure 17(b) shows the initial and equilibrium $\phi$ profiles across the center of the droplet along the $x$ direction with the equilibrium densities as the initial densities in the two phases; from this figure, we can find that in the steady state the $\phi$ change in the two phases is much smaller than the previous case, which further proved our analysis. The small density variations in Fig. 17(b) and the slight $\phi$ conversion between the liquid and gas phases at the beginning stage of the simulation as shown in Figs. 13 and 14 are believed to come from the slight force imbalance at the beginning of the simulation due to the over-predicted surface tension effect by the sharp gradient in the interface and from the numerical errors like the existence of artificial velocity field.

\section{The effect of initial densities in the open gas channel}

Open boundaries such as inlets and outlets essentially share practical and theoretical interests in many situations. For these open flows, it is common to assign a velocity profile or a constant flux at the inlet and meanwhile assign the

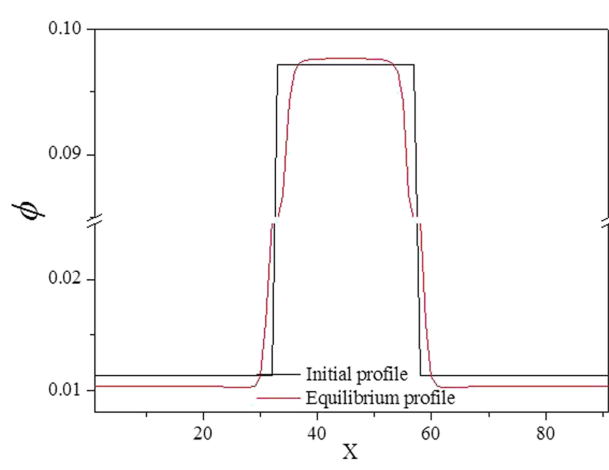

(a)

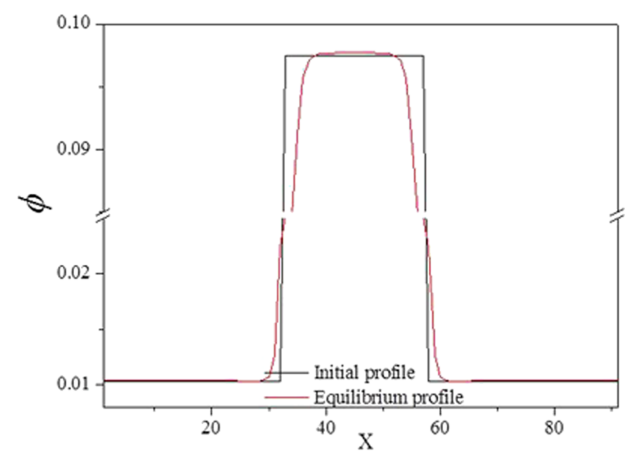

(b)
FIG. 17. The initial and the equilibrium $\phi$ profiles across the center of the droplet along the $x$ direction. (a) Initial densities from the saturation points. (b) Initial densities from the equilibrium state. 


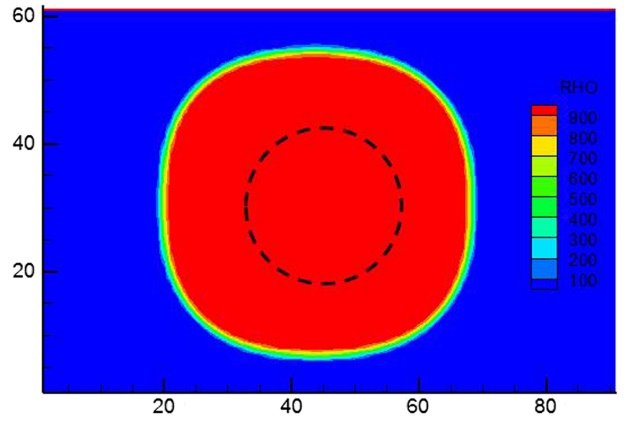

(a)

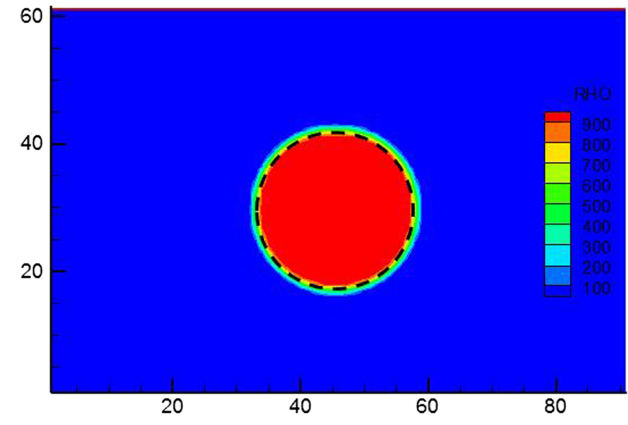

(b)
FIG. 18. Density distributions of the two-phase flow system at time step $=5 \times 10^{6}$ in an open gas channel with different initial $\phi$ settings in the two phases. (a) Initial $\phi$ in the two phases from the saturation points. (b) Initial $\phi$ in the two phases from equilibrium state. free-out boundary condition (the zero-gradient boundary condition or the no-flux condition normal to the wall boundary condition) at the outlet. To investigate the effect of initial densities on the simulation results of a two-phase flow system with a curved interface in an open system, the same gas channel and two-phase assignment as adopted as in the closed system are adopted, but with the 0 flux boundary or the uniform 0 velocity boundary assigned at the inlet and the free-out boundary condition adopted at the outlet, for simplicity.

It is found that the effect of initial densities on the simulation results is even stronger in the open gas channel than in the closed gas channel. To have a clearer observation, the computational time period in the open gas channel case is enlarged to $5 \times 10^{6}$ steps, which is 10 times longer than the period performed in the closed gas channel. Figure 18 shows the density $\rho$ 's distributions of the two-phase flow system with a large density ratio in the open gas channel at the end of the simulation when the simulations are performed with the initial densities $(\phi)$ from the saturation points [Fig. 18(a)] and the equilibrium state [Fig. 18(b)] separately. The dotted black iso-surface shown in Fig. 18 is the expected droplet volume in the equilibrium state. From these figures, it is found that the volume of droplet would expand drastically when the initial densities in the two phases come from saturation points; meanwhile the volume of droplet would basically remain the same or only expand slightly when the initial densities come from the equilibrium state. Figure 19 is the evolution histories of the total $\phi$, the $\phi$ in the liquid and gas phases of the whole domain when the two types of initial densities settings are adopted. From Fig. 19(a), it is found that the total $\phi$ and the $\phi$ in the liquid phase in the whole domain increased rapidly in the whole simulation process with almost the same ratio, and the $\phi$ in the gas phase is simultaneously decreasing but with an obviously slower rate when the initial densities are from the saturation points. Meanwhile, when the initial densities in the liquid and

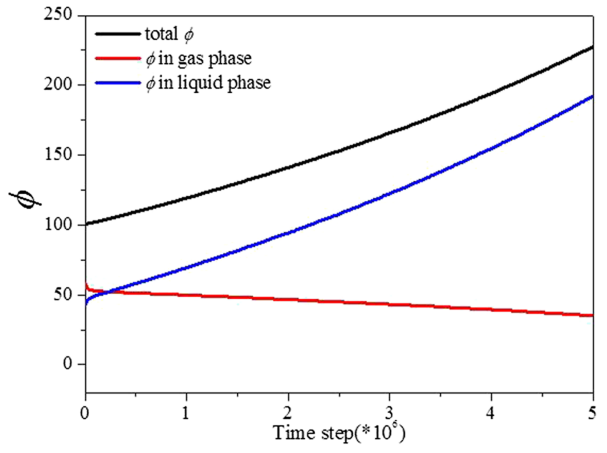

(a)

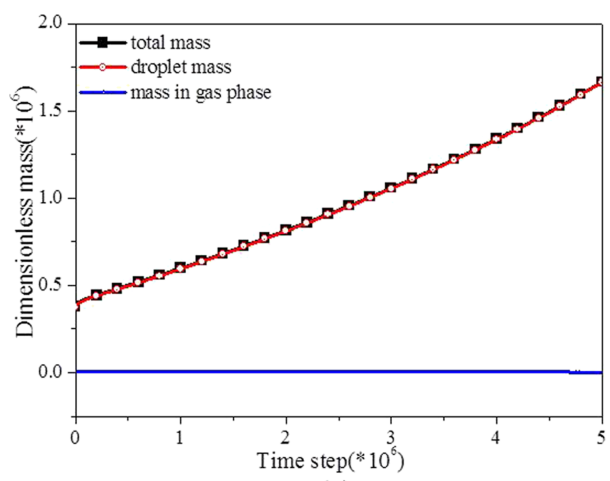

(a)

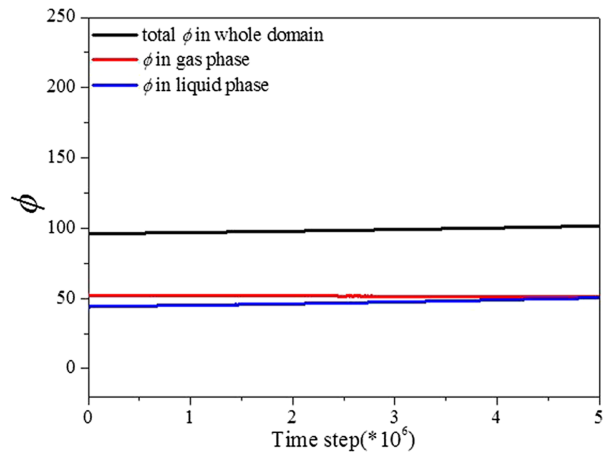

(b)

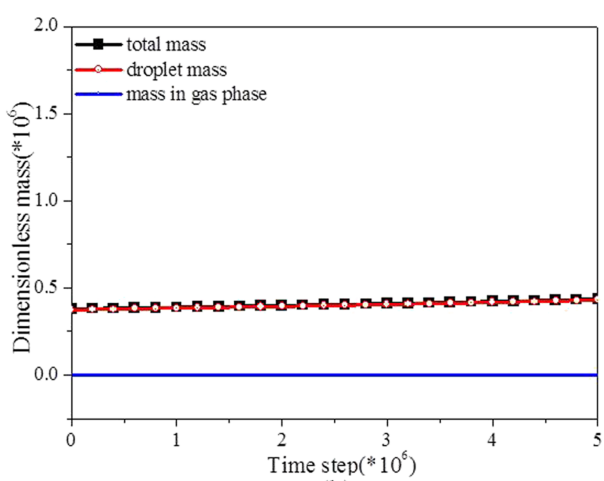

(b)
FIG. 19. Evolution histories of the mass in the small density ratio system in the whole domain of the open gas channel with different initial $\phi$ settings in the two phases. (a) Initial $\phi$ in the two phases from the saturation points. (b) Initial $\phi$ in the two phases from the equilibrium state.
FIG. 20. Evolution histories of the mass in the large density ratio system in the whole domain of the open gas channel with different initial $\phi$ settings in the two phases. (a) Initial $\phi$ in the two phases from the saturation points. (b) Initial $\phi$ in the two phases from the equilibrium state. 


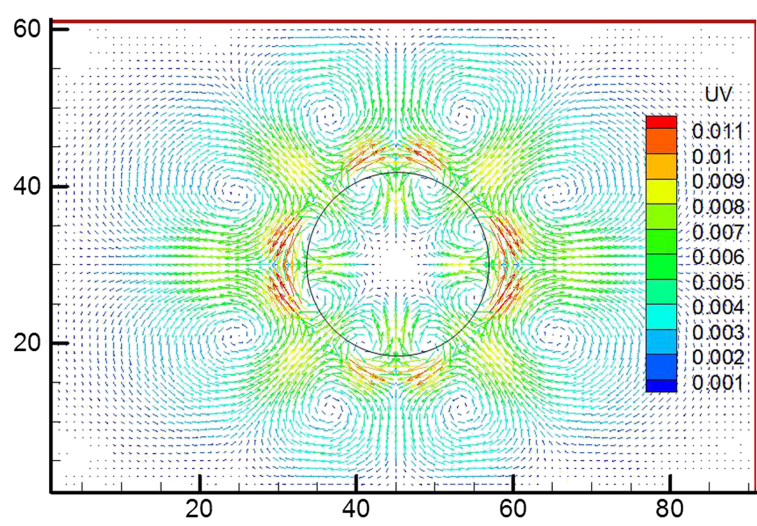

FIG. 21. A typical artificial velocity field observed in the simulation.

gas phases are from the equilibrium state, though the total $\phi$ and the $\phi$ in the liquid phase still continuously increase with almost the same ratio, their increase ratio is much smaller than the previous case; the $\phi$ in the gas phase will basically remain the same amount. The faster increasing ratio of the $\phi$ in the liquid phase than the decreasing ratio of the $\phi$ in the gas phase in the two cases of Fig. 19 proves that the mass increase in the liquid phase is not only from the phase conversion from the gas phase but also from other sources. Figure 20 shows the corresponding evolution histories of the total mass, the mass in the liquid and gas phases described by the density $\rho$; similar tendencies as these in Fig. 19 can be observed.

By comparing the simulation results of a static droplet in an open gas channel with the initial densities from the saturation points and the equilibrium state, it proves that the inappropriate initial densities setting in the liquid and gas phases is the reason for the rapid increase of volume and mass in the simulation. And by comparison of the simulation results of a static droplet in the closed gas channel and the open channel, it proves that the mass and volume expansion problem essentially comes from the open boundaries because in the closed gas channel, no matter which initial densities settings are adopted, the volume and mass of the droplet would remain constant in the steady stage as shown in the figures of Subsection III B. So the next two questions to be answered are how the open boundaries induce the mass increase and volume expansion problem and why the simulation in the open system with initial densities from the saturation points leads to the rapid mass increase and volume expansion problem.

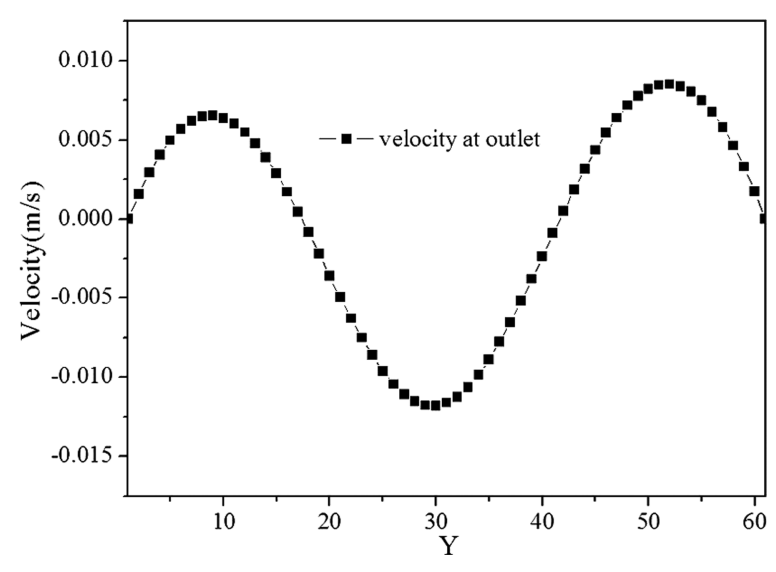

FIG. 22. Example of velocity component distribution along the $x$ direction at the outlet in the steady stage.

Physically speaking, for an open gas channel with uniform 0 velocity profile (or 0 flux boundary) at inlet and free-out boundary at outlet, it corresponds to an open system which allows mass exchange with outside at outlet and does not allow mass exchange with outside at inlet and the solid walls. In the numerical simulation, owing to the existence of numerical error, for example, the artificial velocity field induced around the interface at outlet mass may be exchanged with outside by the advection effect. Figure 21 shows a typical artificial velocity field observed in the simulation and Fig. 22 shows the corresponding velocity distribution at the outlet along the $\mathrm{x}$ direction in the steady stage. So people usually consider that the mass input in the simulation comes from outlet. However, if we carefully check the mass exchanged at the open boundaries, we could find the surprising fact that the outlet is not the only source of mass input but also the inlet. Figure 23 shows the evolution histories of mass (expressed by the order parameter $\phi$ ) input from both the inlet and outlet and their comparison with the total mass in the whole domain under the two types of initial density settings. From the two figures, it can be found that the mass input from the inlet plays the major role in the mass increase of the whole domain, particularly when the simulation is performed with initial densities from the saturation points. And in both the two cases, the contribution of mass input from the outlet is small and keeps the same level, which proves that mass increase of the whole domain eventually comes from the inappropriate initial densities settings at the inlet. As analyzed in Subsection III B, the mass

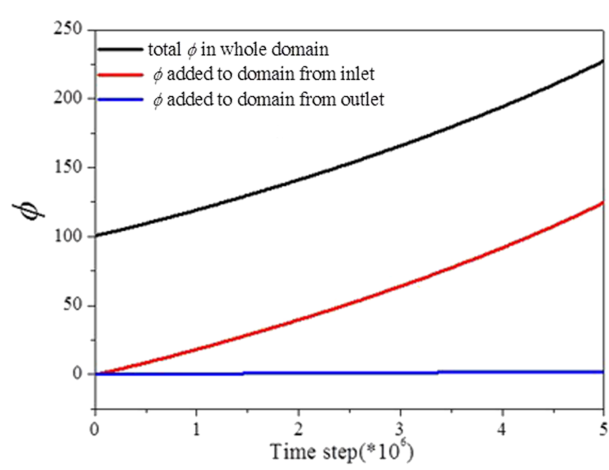

(a)

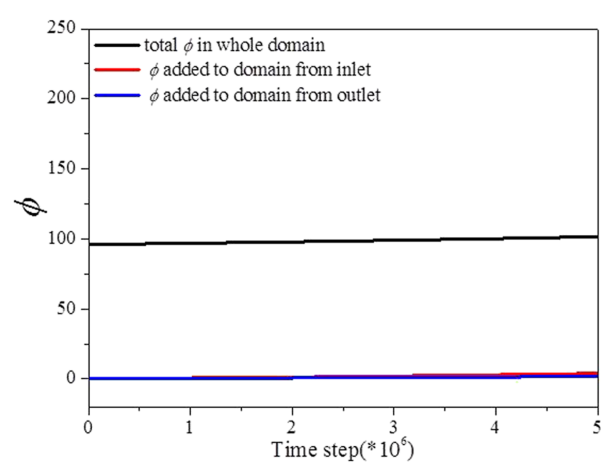

(b)
FIG. 23. Evolution histories of accumulated mass in the small density ratio system added to the domain from inlet and outlet and their comparison with the total mass in the whole domain. (a) Initial $\phi$ in the two phases from the saturation points. (b) Initial $\phi$ in the two phases from equilibrium state. 


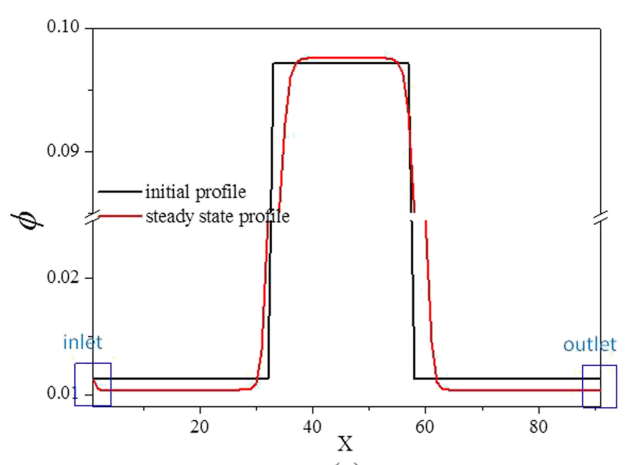

(a)

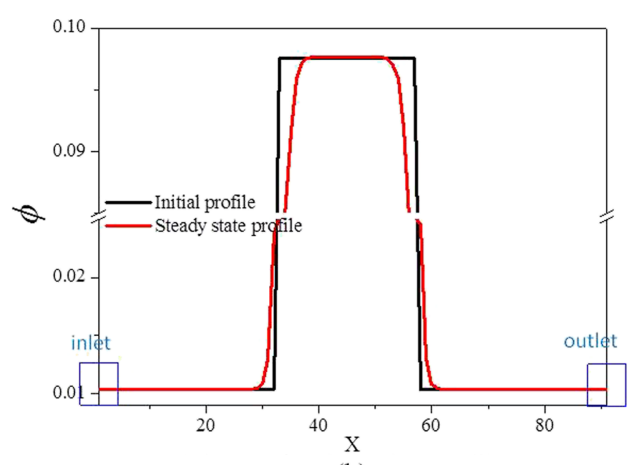

(b)
FIG. 24. $\phi$ profiles across the center of the micro-channel along the $x$ direction in the initial and the beginning of the steady stages (time step $=0$ and 0.5 $\times 10^{6}$ ). (a) Initial densities from the saturation points. (b) Initial densities from the equilibrium state. in the gas phase of the domain would be absorbed into the interface region when the initial densities in the two phases come from the saturation points, owing to the curvature effect of the surface tension force. However, at the inlet of an open gas channel with a constant flux boundary condition or the constant velocity profile boundary condition, at the beginning of every time step, both the constant density and the constant velocity profile are assigned at the inlet lattice points. Figure 24 illustrates the $\phi$ profiles across the center of the droplet along the $\mathrm{x}$ direction at the initial and the beginning of the steady stage (time step $=0.5 \times 10^{6}$ ) under the two types of initial densities settings. As shown in Fig. 24(a) and compared with the case in the closed system shown in Fig. 17(a), the constant inlet density setting makes the inlet a source of mass when the two-phase flow system is moving to the equilibrium state under the curvature effect of surface tension effect, as the density $(\phi)$ reduction arising from the absorption of the gas phase mass into the interface region at the inlet points at the end of each time step would be supplemented with the constant saturation density at the beginning of next time step. When the equilibrium density $(\phi)$ is assigned at the inlet, as there is no or very little difference between the densities at the end and beginning of each time step at the inlet, very little mass would be added to the domain from the inlet, as shown in Fig. 24(b), and the density distribution of $\phi$ at the inlet points and gas phase would remain the same level at the initial and the steady stage. So eventually we can conclude that the volume and mass increasing problem observed in the open system comes from the inappropriate initial densities settings in the gas and liquid phases and is enhanced at the inlet due to the constant density setting.

In fact, for a two-phase flow in an open system, except for the velocity or constant flux boundary condition, the pressure boundary condition is also a common boundary condition adopted at the inlet and outlet. In the one-component-twophase LBM models or the LBM models for non-ideal fluids, the constant pressure at the boundary means the constant density at the boundary, according to Eq. (9) and Fig. 6. So the volume and mass increasing problem reported in this subsection under the constant flux boundary may also be encountered by a two-phase flow system under the pressure boundary condition, and special care should be paid in this situation to avoid obvious numerical errors. Moreover, as discussed in this paper, the two-phase flow system with a small density ratio described by the $f$ i equation is slightly compressible, which is similar to the two-phase flow systems described by the other LBM models, such as the one-component-two-phase model of pseudopotential model ( $\mathrm{S}-\mathrm{C}$ model) and the kinetic theory based LBM. So the problem and solution reported in this paper are also general in those models although sometimes the equations of state (EOS) adopted in these LBM models are different, for example, in the kinetic theory based model, the CarnahanStarling equation of state is used instead of the van der Waals equation.

The initial density related numerical phenomena reported in this paper have been proven to arise from the curvature effect of surface tension and the slight compressibility of the LBM. The curvature of a 2D droplet indeed is the curvature of a 3D column. In the 3D cases, the curvature effect of a droplet with the same radius will have a much stronger surface tension effect, which will lead to stronger mass and volume variation problems if the initial densities in the two phases are not carefully set up. ${ }^{39}$ So the recommendation of adopting the equilibrium densities as the initial densities in the two phases is not only limited in the $2 \mathrm{D}$ simulation but also in the $3 \mathrm{D}$ simulation.

\section{CONCLUSION}

1. The volume and mass variation problem observed in the simulation of a static droplet in a closed gas channel is proven to come from the adoption of the saturation densities in the liquid and gas phases as the initial densities and such a problem can be removed by adopting the initial densities from the equilibrium state.

2. The volume and mass variation problem observed in the simulation of a static droplet in an open gas channel with constant flux inlet and free-out outlet boundary condition is proven to also come from the adoption of the saturation densities in the liquid and gas phases as the initial densities, and it is enhanced by the constant density setting at the inlet. Such a problem can also be removed greatly by adopting the initial densities from the equilibrium state.

\section{ACKNOWLEDGMENTS}

The authors acknowledge the financial support of JSPS KAKENHI Grant No. 15H03912 from the Japan Society for the Promotion of Science (JSPS). 
${ }^{1}$ Z. Guo, C. Zheng, and B. Shi, "Force imbalance in lattice Boltzmann equation for two-phase flows," Phys. Rev. E 83(3), 036707 (2011).

${ }^{2} \mathrm{~K}$. Connington and T. Lee, "A review of spurious currents in the lattice Boltzmann method for multiphase flows," J. Mech. Sci. Technol. 26(12), 3857-3863 (2012).

${ }^{3}$ M. P. Allen, "Introduction to molecular dynamics simulation," in Computational Soft Matter: From Synthetic Polymers to Proteins (John von Neumann Institute for Computing, Jülich, 2004), Vol. 23, pp. 1-28.

${ }^{4}$ C. W. Hirt and B. D. Nichols, "Volume of fluid (VOF) method for the dynamics of free boundaries," J. Comput. Phys. 39(1), 201-225 (1981).

${ }^{5}$ D. Gueyffier, J. Li, A. Nadim, R. Scardovelli, and S. Zaleski, "Volumeof-fluid interface tracking with smoothed surface stress methods for threedimensional flows," J. Comput. Phys. 152(2), 423-456 (1999).

${ }^{6}$ J. A. Sethian and P. Smereka, "Level set methods for fluid interfaces," Annu. Rev. Fluid Mech. 35(1), 341-372 (2003).

${ }^{7}$ S. Osher and R. P. Fedkiw, "Level set methods: An overview and some recent results," J. Comput. Phys. 169(2), 463-502 (2001).

${ }^{8}$ T. Yabe, F. Xiao, and T. Utsumi, "The constrained interpolation profile method for multiphase analysis," J. Comput. Phys. 169(2), 556-593 (2001).

${ }^{9}$ T. Yabe, Y. Ogata, K. Takizawa, T. Kawai, A. Segawa, and K. Sakurai, "The next generation CIP as a conservative semi-Lagrangian solver for solid, liquid and gas," J. Comput. Appl. Math. 149(1), 267-277 (2002).

${ }^{10}$ A. A. Mohamad, Lattice Boltzmann Method: Fundamentals and Engineering Applications with Computer Codes (Springer Science \& Business Media, 2011).

${ }^{11}$ H. Huang, M. Sukop, and X. Lu, Multiphase Lattice Boltzmann Methods: Theory and Application (John Wiley \& Sons, 2015).

${ }^{12} \mathrm{~S}$. Succi, The Lattice-Boltzmann Equation for Fluid Dynamics and Beyond (Oxford University Press, Oxford, 2001).

${ }^{13}$ M. C. Sukop and D. T. Thorne, Jr., Lattice Boltzmann Modeling: An Introduction for Geoscientists and Engineers (Springer Berlin Heidelberg, Berlin, Heidelberg, 2006).

${ }^{14}$ S. Leclaire, N. Pellerin, M. Reggio, and J.-Y. Trépanier, "A multiphase lattice Boltzmann method for simulating immiscible liquid-liquid interface dynamics," Appl. Math. Modell. 40(13-14), 6376-6394 (2016).

${ }^{15}$ A. K. Gunstensen, D. H. Rothman, S. Zaleski, and G. Zanetti, "Lattice Boltzmann model of immiscible fluids," Phys. Rev. A 43(8), 4320 (1991).

${ }^{16}$ D. H. Rothman and J. M. Keller, "Immiscible cellular-automaton fluids," J. Stat. Phys. 52(3-4), 1119-1127 (1988).

${ }^{17} \mathrm{X}$. Shan and H. Chen, "Lattice Boltzmann model for simulating flows with multiple phases and components," Phys. Rev. E 47(3), 1815-1819 (1993).

${ }^{18} \mathrm{X}$. Shan and H. Chen, "Simulation of nonideal gases and liquid-gas phase transitions by the lattice Boltzmann equation," Phys. Rev. E 49(4), 29412948 (1994).

${ }^{19}$ M. R. Swift, W. R. Osborn, and J. M. Yeomans, "Lattice Boltzmann simulation of nonideal fluids," Phys. Rev. Lett. 75(5), 830-833 (1995).

${ }^{20}$ M. R. Swift, E. Orlandini, W. R. Osborn, and J. M. Yeomans, "Lattice Boltzmann simulations of liquid-gas and binary fluid systems," Phys. Rev. E 54(5), 5041-5052 (1996).

${ }^{21}$ X. He, X. Shan, and G. D. Doolen, "Discrete Boltzmann equation model for nonideal gases," Phys. Rev. E 57(1), R13-R16 (1998).
${ }^{22}$ J. W. Cahn and J. E. Hilliard, "Free energy of a nonuniform system. I. Interfacial free energy," J. Chem. Phys. 28(2), 258-267 (1958).

${ }^{23}$ T. Lee and C.-L. Lin, "A stable discretization of the lattice Boltzmann equation for simulation of incompressible two-phase flows at high density ratio," J. Comput. Phys. 206(1), 16-47 (2005).

${ }^{24}$ Y. Tabe, Y. Lee, T. Chikahisa, and M. Kozakai, "Numerical simulation of liquid water and gas flow in a channel and a simplified gas diffusion layer model of polymer electrolyte membrane fuel cells using the lattice Boltzmann method," J. Power Sources 193(1), 24-31 (2009).

${ }^{25} \mathrm{~K}$. Moriyama and T. Inamuro, "Lattice Boltzmann simulations of water transport from the gas diffusion layer to the gas channel in PEFC," Commun. Comput. Phys. 9, 1206-1218 (2011).

${ }^{26}$ Y. Ben Salah, Y. Tabe, and T. Chikahisa, "Two phase flow simulation in a channel of a polymer electrolyte membrane fuel cell using the lattice Boltzmann method," J. Power Sources 199(0), 85-93 (2012).

${ }^{27}$ S. Succi, The Lattice Boltzmann Equation: For Fluid Dynamics and Beyond (Oxford University Press, 2001).

${ }^{28} \mathrm{~T}$. Inamuro, N. Konishi, and F. Ogino, "A Galilean invariant model of the lattice Boltzmann method for multiphase fluid flows using free-energy approach," Comput. Phys. Commun. 129(1-3), 32-45 (2000).

${ }^{29}$ Q. Li, K. H. Luo, Y. J. Gao, and Y. L. He, "Additional interfacial force in lattice Boltzmann models for incompressible multiphase flows," Phys. Rev. E 85(2), 026704 (2012).

${ }^{30}$ M. C. Sukop and D. Or, "Invasion percolation of single component, multiphase fluids with lattice Boltzmann models," Phys. B 338(1-4), 298-303 (2003).

${ }^{31}$ X. He, S. Chen, and R. Zhang, "A lattice Boltzmann scheme for incompressible multiphase flow and its application in simulation of Rayleigh-Taylor instability," J. Comput. Phys. 152(2), 642-663 (1999).

${ }^{32}$ T. Inamuro, T. Ogata, S. Tajima, and N. Konishi, "A lattice Boltzmann method for incompressible two-phase flows with large density differences," J. Comput. Phys. 198(2), 628-644 (2004).

${ }^{33}$ M. Yoshino and Y. Mizutani, "Lattice Boltzmann simulation of liquid-gas flows through solid bodies in a square duct," Math. Comput. Simul. 72(2-6), 264-269 (2006).

${ }^{34}$ P. Yuan and L. Schaefer, "Equations of state in a lattice Boltzmann model," Phys. Fluids 18(4), 042101 (2006).

${ }^{35} \mathrm{~J}$.-m. Gong, N. Oshima, and Y. Tabe, "Reduction of spurious velocity in the free-energy-based lattice Boltzmann method for large density ratio," J. Therm. Sci. Technol. 10(1), JTST0004 (2015).

${ }^{36}$ J. Gong, N. Oshima, and Y. Tabe, "Spurious velocity from the cutoff and magnification equation in free energy-based LBM for two-phase flow with a large density ratio," Comput. Math. Appl. (published online 2016).

${ }^{37}$ Q. Lou, Z. Guo, and C. Zheng, "Some fundamental properties of lattice Boltzmann equation for two phase flows," Comput. Model. Eng. Sci. 76(3), 175-188 (2011).

${ }^{38}$ D. P. Ziegler, "Boundary conditions for lattice Boltzmann simulations," J. Stat. Phys. 71(5), 1171-1177 (1993).

${ }^{39}$ J. Gong, N. Oshima, and Y. Tabe, "Effect of free-out outlet boundary condition on the free energy based two-phase flow LBM with large density ratio," in Mechanical Engineering Congress, Japan 2014 (The Japan Society of Mechanical Engineers (JSME), 2014), pp. G0110103-1-G0110103-5; see http://ci.nii.ac.jp/naid/110009943209. 